\title{
Migraine and cluster headache show impaired neurosteroids patterns
}

\author{
Angela Koverech 1,2,10, Claudia Cicione ${ }^{3}$, Luana Lionetto ${ }^{3,9}$, Marta Maestri ${ }^{1}$, Francesco Passariello ${ }^{1}$, \\ Elisabetta Sabbatini ${ }^{1}$, Matilde Capi ${ }^{4,9}$, Cristiano Maria De Marco ${ }^{5}$, Martina Guglielmetti ${ }^{5,6}$, Andrea Negro ${ }^{1,5}$, \\ Luisa Di Menna ${ }^{7}$, Maurizio Simmaco ${ }^{8,9}$, Ferdinando Nicoletti ${ }^{7,10}$ and Paolo Martelletti ${ }^{1,2,5^{*}}$ (D)
}

\begin{abstract}
Background: Perturbation of neuronal excitability contributes to migraine. Neurosteroids modulate the activity of $Y$ aminobutyric acid $\mathrm{A}$ and $\mathrm{N}$-methyl-d-aspartate receptors, and might be involved in the pathogenesis of migraine. Here, we measured plasma levels of four neurosteroids, i.e., allopregnanolone, epiallopregnanolone, dehydroepiandrosterone and deydroepiandrosterone sulfate, in patients affected by episodic migraine, chronic migraine, or cluster headache.

Methods: Nineteen female patients affected by episodic migraine, 51 female patients affected by chronic migraine, and 18 male patients affected by cluster headache were recruited to the study. Sex- and age-matched healthy control subjects (31 females and 16 males) were also recruited. Patients were clinically characterized by using validated questionnaires. Plasma neurosteroid levels were measured by liquid chromatography-tandem mass spectrometry.

Results: We found disease-specific changes in neurosteroid levels in our study groups. For example, allopregnanolone levels were significantly increased in episodic migraine and chronic migraine patients than in control subjects, whereas they were reduced in patients affected by cluster headache. Dehydroepiandrosterone and dehydroepiandrosterone sulfate levels were reduced in patients affected by chronic migraine, but did not change in patients affected by cluster headache.

Conclusion: We have shown for the first time that large and disease-specific changes in circulating neurosteroid levels are associated with chronic headache disorders, raising the interesting possibility that fluctuations of neurosteroids at their site of action might shape the natural course of migraine and cluster headache. Whether the observed changes in neurosteroids are genetically determined or rather result from exposure to environmental or intrinsic stressors is unknown. This might also be matter for further investigation because stress is a known triggering factor for headache attacks in both migraineurs and cluster headache patients.
\end{abstract}

Keywords: Episodic migraine, Chronic migraine, Cluster headache, Allopregnanolone, Dehydroepiandrosterone

\section{Introduction}

Migraine is a chronic disease with relevant clinical and social implications. The recently released results of the Global Burden of Disease initiative reveal that migraine is the fifth leading chronic disease on the planet, being more prevalent than diabetes [1,2]. Also, migraine negatively influences quality of life, and is the fourth leading

\footnotetext{
* Correspondence: paolo.martelletti@uniroma1.it

${ }^{1}$ Department of Clinical and Molecular Medicine, Sapienza University and Regional Referral Headache Centre, Sant'Andrea Hospital, via di Grottarossa 1035-1039, 00189 Rome, Italy

${ }^{2}$ Residency Program of Internal Medicine, School of Medicine and Psychology, Sapienza University, 00189 Rome, Italy

Full list of author information is available at the end of the article
}

disease per number of years lived with disability [1, 2]. These figures highlight the existing gaps in the preventive and symptomatic treatment of migraine.

Among other predisposing factors, the pathogenesis of migraine and cluster headache appears to be related to an impaired control of neuronal excitability [3]. Hence, unraveling the trans-synaptic and/or intracellular mechanisms that alter neuronal firing in migraine and cluster headache may provide new insights into the pathophysiology of these disorders and pave the way to novel therapeutic interventions. Neurosteroids are endogenous steroids synthesized in the central nervous system (CNS) that modulate neuronal excitability by interacting with 
either $\gamma$-aminobutyric acid A or N-methyl-d-aspartate receptors [4-6]. Neurosteroids are synthesized in glial cells from cholesterol or other precursors via progressive reduction of the A-ring of the steroid molecule [4]. Most of the current research on neurosteroids focuses on allopregnanolone (AP), epiallopregnanolone (EAP), dehydroepiandrosterone (DHEA), and DHEA sulfate (DHEAS). AP-like neurosteroids are potent positive allosteric modulators as well as direct activators of both synaptic and extrasynaptic $\gamma$-aminobutyric acid A receptors, and, therefore, they can maximally enhance synaptic phasic inhibition and extrasynaptic tonic inhibition [4]. In contrast, DHEA and DHEAS are weak antagonists of $\gamma$-aminobutyric acid A receptor and agonists of $\mathrm{N}$-methyl-d-aspartate receptors [7]. Thus, allopregnanolone and DHEA/DHEAS display opposite effects on neuronal excitability. The clinical relevance of neurosteroids is exemplified by their potential role in the pathophysiology of epilepsy [8, 9]. An imbalance between excitatory and inhibitory neurotransmission leading to a hyperactivation of $\mathrm{N}$-methyl-d-aspartate receptors is considered as a key event in the development of migraneous aura and in mechanisms of nociceptive sensitization underlying the central component of migraneous pain $[10,11]$. Owing to their mechanisms of action, neurosteroids might participate to the pathophysiology of migraine particularly under conditions associated with changes in the peripheral production of progesterone and other neurosteroid precursors (e.g., the pre-menstraul period). The association between serum $\gamma$-aminobutyric acid levels and the clinical characteristics of migraine suggests a causal link between changes in $\gamma$-aminobutyric acid mediated neurotransmission and the pathophysiology of migraine [12]. However, whether neurosteroids are part of this link remains undetermined.

The aim of our study was to measure for the first time circulating levels of neurosteroids in patients with ICHD3-beta confirmed diagnosis [13] of episodic or chronic migraine (EM, CM), medication overuse headache $(\mathrm{MOH})$ and cluster headache $(\mathrm{CH})$, and to explore possible associations with their clinical characteristics.

Migraine and cluster headache are complex and multifaceted clinical diseases, whose conceptual framework has been recently organized by the International Classification of Headache Disorders 3rd Edition beta classification [13]. In particular, migraine is considered as a primary headache and patients are diagnosed as having episodic (EM) or chronic migraine (CM) according to well established clinical parameters [13]. In the International Classification of Headache Disorders 3rd Edition beta classification, medication-overuse headache $(\mathrm{MOH})$ is considered within secondary headaches and thus it is independent from migraine [14]. However, it has been recently proposed that $\mathrm{MOH}$ should be more correctly considered a complication of $\mathrm{CM}$, if not even in some cases its natural evolution as $\mathrm{CM}$ plus medication overuse $(\mathrm{CM}+\mathrm{MO})[15,16]$. Cluster headache $(\mathrm{CH})$ is also a primary headache, but it is not classified together with migraine rather as a trigeminal autonomic cephalalgia [13].

\section{Materials and methods}

The protocol has been devised as a cross-sectional study. It complies with the rules set by the Declaration of Helsinki and subsequent amendments, and has been approved by the Azienda Ospedaliero-Universitaria Sant'Andrea Ethics Committee. The study has been carried out between February and December 2017.

\section{Patients}

Patients of both sexes, aging $18-80$ years and referring to the Headache Unit at Azienda Ospedaliera-Universitaria S. Andrea, Rome, were recruited for the study. Patients were cognitively able to sign the informed consent, and received the diagnosis of $\mathrm{EM}$, or $\mathrm{CM}$, or $\mathrm{CM}+\mathrm{MO}$, or $\mathrm{CH}$ according to the International Classification of Headache Disorders 3rd Edition -beta criteria [13]. Patients with cancer, liver failure, renal failure or recent administration of benzodiazepines were excluded from the study. Sex- and age-matched healthy subjects were recruited as controls.

\section{Study design}

After signing the informed consent, patients were enrolled and the following information collected and registered: age, sex, education, comorbidities, actual drug therapy, the presence of a concurrent migraine/headache attack, number of attacks/month, day of the menstrual cycle (women only). Then, validated psychometric and functional questionnaires were provided. In particular, patients were asked to fill the Beck Depression Inventory (BDI), the Migraine Disability Assessment Test (MIDAS; only migraine patients), the Headache Impact test (HIT-6), and the Self-rating Anxiety Scale (SAS). BDI is a validated questionnaire for quantifying the severity of depression. MIDAS provides a precise assessment of migraine-induced disability. HIT-6 measures the impact of headache on daily activities. SAS provides a quantitative assessment of anxiety.

\section{Biochemistry}

After signing informed consent, blood sample was collected, and immediately centrifuged. This occurred in the morning, between $8 \mathrm{AM}$ and $9 \mathrm{AM}$. Aliquots of plasma were separated and kept at $-20^{\circ} \mathrm{C}$ until further analysis.

Circulating levels of AP $(\mathrm{ng} / \mathrm{mL})$, EAP $(\mathrm{ng} / \mathrm{mL})$, DHEA $(\mathrm{ng} / \mathrm{mL})$ and DHEAS $(\mu \mathrm{g} / \mathrm{mL})$ were measured by liquid chromatography-tandem mass spectrometry (LC-MS/ 
MS), using the protocol developed and validated at our institution and previously described [17]. Briefly, the analytes were first derivatized with 2-hydrazinopyridine and extracted from plasma using solid phase extraction. Then, the compounds were separated and detected by LC-MS/MS. A mobile phase of formic acid $(0.1 \%)$ in water and methanol through a gradient of composition and a flow rate of $0.3 / \mathrm{mL} / \mathrm{min}$ was used since we demonstrated that results in good separations of the analytes [17]. By using this protocol, linear responses in wide range of concentrations and limits of quantification ranging from 10 (DHEAS) to $40 \mathrm{pg} / \mathrm{mL}$ (DHEA) are obtained in $<9$ min [17].

\section{Statistical analysis}

Results were statistically analysed by Student's t test and by one-way ANOVA and Fisher's LSD as a post-hoc test where appropriate. Possible associations between continuous variables were assessed by the Pearson's correlation test. Data are presented as means \pm S.D.

\section{Results}

Screening and enrolment procedures are illustrated by the CONSORT flow diagram in Fig. 1.

Considering the imbalanced gender distribution within groups, and the potential role of female sex hormones as precursors of neurosteroids [18], we decided to consider only female patients for $\mathrm{EM}, \mathrm{CM}$ and $\mathrm{MOH}$, and only male patients for $\mathrm{CH}$. Also, as previously mentioned $[15,16]$, $\mathrm{MOH}$ can be considered as a complication or even as the natural evolution of CM. Therefore, patients with $\mathrm{MOH}$ were merged into the CM group.

The control groups included 31 female healthy individuals and 16 male healthy individuals. Patients' and controls' characteristics are reported in Table 1.

\section{Episodic migraine}

Plasma neurosteroid levels fell within the expected range in all our patients and controls (e.g., 3-10 nM corresponding approximately to $1-3.7 \mathrm{ng} / \mathrm{ml}$ of AP) [19]. $\mathrm{AP}$ and EAP levels were largely increased in patients affected by EM, whereas DHEA levels were reduced by $>40 \%$ (Table 2). No changes in DHEAS levels and in the ratio between $\mathrm{AP}$ and EAP were found in patients affected by EM with respect to healthy controls $(1.9 \pm$ 0.9 vs $1.8 \pm 1.2 ; \mathrm{p}=$ n.s.). Knowing that the ovarian cycle has a strong impact on neurosteroid synthesis [16], we also examined AP, EAP, DHEA, and DHEAS levels in pre- and post-menopausal patients and healthy controls. Differences in AP, EAP, and DHEAS levels between EM patients and controls were maintained when subjects were stratified according to their menopausal status. In addition, no changes in neurosteroid levels were found between pre- and post-menopausal EM patients.

No significant associations were found between blood neurosteroids and BDI, SAS, HIT-6 and MIDAS scores, and educational level in EM patients and controls.

\section{Chronic migraine (including medication-overuse)}

Circulating levels of neurosteroids in CM patients are shown in Table 3. Similarly to EM patients, CM patients showed large increases in AP levels, whereas EAP levels did not change, and levels of both DHEA and DHEAS were reduced. A direct comparison of AP, DHEA, and DHEAS levels between patients affected by EM and CM is shown in Table 4. Interestingly, DHEA and DHEAS levels were significantly lower in $\mathrm{CM}$ patients with respect to EM patients, whereas AP levels did not differ between the two groups (Table 4). Thus, neurosteroids behaved differently in EM and CM patients, as compared to their respective controls. At least in $\mathrm{CM}$ patients, changes in neurosteroid levels were unaffected by the hormonal status, as shown by a subgroup analysis in patients examined before and after menopause, or in fertile patients examined in the follicular and luteal phases of the ovarian cycle (data not shown). Thus, it appears that migraine itself rather than fluctuations in

\begin{tabular}{|c|c|c|c|c|}
\hline \multirow{4}{*}{ Assessed for eligibility } & Episodic migraine & \begin{tabular}{|l|} 
Chronic migraine \\
\end{tabular} & Medication-overuse headache & Cluster headache \\
\hline & $\downarrow$ & $\downarrow$ & $\downarrow$ & $\downarrow$ \\
\hline & 22 & 31 & 35 & 19 \\
\hline & $\downarrow$ & $\downarrow$ & $\downarrow$ & $\downarrow$ \\
\hline \multirow[t]{4}{*}{ Excluded } & $3 M$ & $3 \mathrm{M}, 4 \mathrm{decl}$ & 3M, 5 decl. & $1 \mathrm{~F}$ \\
\hline & $\downarrow$ & $\downarrow$ & $\downarrow$ & $\downarrow$ \\
\hline & 19 & 24 & 27 & 18 \\
\hline & $\downarrow$ & $\downarrow$ & $\downarrow$ & $\downarrow$ \\
\hline $\begin{array}{l}\text { Analysed } \\
\text { Fig. } 1 \text { CONSORT flow diagram of the st }\end{array}$ & $\begin{array}{c}19 \\
\text { udy }(M=\text { males; }\end{array}$ & 24 decl. = declin & 27 & 18 \\
\hline
\end{tabular}


Table 1 Demographic and clinical characteristics of the recruited patients affected by episodic migraine, chronic migraine, or cluster headache, and their respective healthy controls

\begin{tabular}{|c|c|c|c|c|c|}
\hline & Episodic migraine & Chronic migraine $^{a}$ & Controls (female) & Cluster headache & $\overline{\text { Controls (male) }}$ \\
\hline n. & 19 & 51 & 31 & 18 & 16 \\
\hline Age (years) & $41,6 \pm 16,2$ & $51,6 \pm 10,9$ & $52,1 \pm 17,8$ & $46,2 \pm 15,8$ & $58,7 \pm 13,8$ \\
\hline Menopause & $36 \%(7 / 19)$ & $62 \%(32 / 51)$ & $54 \%(17 / 31)$ & N.A. & N.A. \\
\hline Sampling during migraine/headache attack & $0 \%$ & $53 \%$ & N.A. & $39 \%$ & N.A. \\
\hline $\begin{array}{l}\mathrm{BDI}(\mathrm{n} \text {.) } \\
\text { no/mild/mod./severe }\end{array}$ & $\begin{array}{l}18 \\
12 / 4 / 2\end{array}$ & $\begin{array}{l}49 \\
26 / 11 / 7 / 5\end{array}$ & N.A. & $\begin{array}{l}18 \\
14 / 3 / 0 / 1\end{array}$ & N.A. \\
\hline $\begin{array}{l}\text { SAS (n.) } \\
\text { low/moderate/severe }\end{array}$ & $\begin{array}{l}17 \\
14 / 3 / 0\end{array}$ & $\begin{array}{l}48 \\
27 / 18 / 3\end{array}$ & N.A. & $\begin{array}{l}18 \\
16 / 2 / 0\end{array}$ & N.A. \\
\hline $\begin{array}{l}\text { HIT-6 (n.) } \\
\text { mild/mod./relevant/severe }\end{array}$ & $\begin{array}{l}18 \\
5 / 0 / 0 / 13\end{array}$ & $\begin{array}{l}49 \\
1 / 2 / 2 / 44\end{array}$ & N.A. & $\begin{array}{l}18 \\
2 / 5 / 3 / 8\end{array}$ & N.A. \\
\hline $\begin{array}{l}\text { MIDAS (n.) } \\
I \rightarrow N \text { (low } \rightarrow \text { severe disability) }\end{array}$ & $\begin{array}{l}18 \\
2 / 4 / 5 / 7\end{array}$ & $\begin{array}{l}49 \\
4 / 5 / 3 / 37\end{array}$ & N.A. & N.A. & N.A. \\
\hline
\end{tabular}

ovarian hormones, is the main driver of neurosteroid changes in CM patients.

In the overall population of $\mathrm{CM}$ patients neurosteroid levels also did no change when blood samples were collected during a migraine attack or in the interictal period (Table 5). However, changes were found when patients affected by $\mathrm{CM}+\mathrm{MO}$ were isolated from the overall population of CM patients according to the ICDH3-beta diagnostic criteria. In these patients, AP levels were lower during the headache attack $(0.9 \pm 0.2 \mathrm{ng} / \mathrm{mL}$ vs $1.1 \pm 0.2$ $\mathrm{ng} / \mathrm{mL}$, respectively; $p<0.05$ ), whereas in the remaining CM patients, DHEAS levels were higher during the attack $(1.8 \pm 1.4 \mu \mathrm{g} / \mathrm{mL}$ vs $0.9 \pm 0.6 \mu \mathrm{g} / \mathrm{mL}$, respectively; $p<0.05)$.

$\mathrm{AP}$ and EAP are known to differentially regulate $\gamma$-aminobutyric acid A receptors, with AP activating and EAP inhibiting receptor function, respectively [20]. Hence, we measured the AP/EAP ratio as a surrogate marker of the cumulative effects of these neurosteroids on $\gamma$-aminobutyric acid mediated transmission. The AP/ EAP ratio was higher In the overall population of $\mathrm{CM}$ patients with respect to healty control $(3.0 \pm 1.7$ vs $1.8 \pm$ 1.2 , respectively; $p<0.01$ ), whether or not blood samples were collected during the headache attack or in the interictal period. The subpopulation of patients with $\mathrm{CM}+\mathrm{MO}$ showed a trend to a reduction in the AP/EAP ratio during the attack with respect to the interictal period ( $2.2 \pm 1.2$ vs $3.4 \pm 1.8$, respectively; $p=0.06$ ).

Table 2 Plasma neurosteroid levels in patients affected by episodic migraine patients and healthy controls

\begin{tabular}{llll}
\hline & Episodic migraine & Controls & $P$ \\
\hline AP $(\mathrm{ng} / \mathrm{mL})$ & $1.3 \pm 0.5$ & $0.6 \pm 0.3$ & $<0.01$ \\
EAP $(\mathrm{ng} / \mathrm{mL})$ & $0.7 \pm 0.2$ & $0.4 \pm 0.1$ & $<0.01$ \\
DHEA $(\mathrm{ng} / \mathrm{mL})$ & $2.9 \pm 1.5$ & $5.1 \pm 3.8$ & $<0.05$ \\
DHEAS $(\mu \mathrm{g} / \mathrm{mL})$ & $2.4 \pm 1.1$ & $2.7 \pm 2.0$ & n.s. \\
\hline
\end{tabular}

Values are means \pm S.D. Statistical analysis was performed by Student's $t$ test
No significant associations were found between plasma neurosteroid levels and BDI, SAS, HIT-6 or MIDAS scores. In addition, there was no association between neurosteroids and education level in our patients.

\section{Cluster headache}

Patients affected by $\mathrm{CH}$ showed lower levels of AP and no changes in other neurosteroids with respect to healthy controls (Table 6). The reduction in AP levels was maintained when blood samples were collected during or between headache attacks $(0.4 \pm 0.1 \mathrm{ng} / \mathrm{mL} ; n=6$, $p<0.05$ vs controls during attacks, and $0.3 \pm 0.1 \mathrm{ng} / \mathrm{mL}$; $n=12$, vs the same controls, $\mathrm{p}<0.05$ in the interictal period). The other neurosteroids did not change during or between headache attacks.

No significant associations were found between neurosteroids and BDI, SAS or HIT-6 scores or education level also in patients affected by $\mathrm{CH}$.

\section{Discussion}

Our findings suggest that changes in neurosteroid levels contribute to the pathophysiology of migraine and cluster headache, with the obvious limitation that plasma levels may not entirely reflect CNS levels. We found elevated AP levels and reduced DHEA levels in all patients affected by migraine, whereas EAP levels were increased

Table 3 Plasma neurosteroid levels in patients affected by chronic migraine patients and healthy controls

\begin{tabular}{llll}
\hline & $\begin{array}{l}\text { Chronic migraine } \\
\text { (overall population) }\end{array}$ & Controls & $P$ \\
\hline AP $(\mathrm{ng} / \mathrm{mL})$ & $1.1 \pm 0.3$ & $0.61 \pm 0.3$ & $<0.01$ \\
$\operatorname{EAP}(\mathrm{ng} / \mathrm{mL})$ & $0.4 \pm 0.2$ & $0.41 \pm 01$ & n.s. \\
DHEA $(\mathrm{ng} / \mathrm{mL})$ & $1.6 \pm 1.1$ & $5.1 \pm 3.8$ & $<0.01$ \\
DHEAS $(\mu \mathrm{g} / \mathrm{mL})$ & $1.2 \pm 0.9$ & $2.76 \pm 2.0$ & $<0.01$ \\
\hline
\end{tabular}

Values are means \pm S.D. Statistical analysis was performed by Student's $t$ test 
Table 4 Comparative analysis of DHEA, DHEAS, and AP levels in patients affected by episodic or chronic migraine

\begin{tabular}{llll}
\hline & $\begin{array}{l}\text { Episodic migraine } \\
(n=19)\end{array}$ & $\begin{array}{l}\text { Chronic migraine } \\
(n=51)\end{array}$ & $P$ \\
\hline DHEA $(\mathrm{ng} / \mathrm{mL})$ & $2.9 \pm 1.5$ & $1.6 \pm 1.1$ & $<0.01$ \\
DHEAS $(\mu \mathrm{g} / \mathrm{mL})$ & $2.4 \pm 1.1$ & $1.2 \pm 0.9$ & $<0.01$ \\
AP $(\mathrm{ng} / \mathrm{mL})$ & $1.3 \pm 0.5$ & $1.1 \pm 0.3$ & n.s. \\
\hline
\end{tabular}

Values are means + SD. Statistical analysis was performed by Student's t test

only in patients affected by EM, and DHEAS levels were reduced only in patients affected by $\mathrm{CM}$. It is generally believed that abnormalities in the regulation of neuronal excitability underlie the pathophysiology of migraine, giving raise to cortical spreading depression or to functional changes in pain regulatory centers of the brainstem [21]. Neurosteroids play a key role in the regulation of neuronal excitability by modulating the activity of $\gamma$-aminobutyric acid A and N-methyl-d-aspartate receptors [5], with AP behaving as a positive allosteric modulator of both synaptic and extrasynaptic $\gamma$-aminobutyric acid A receptors by interacting with a picrotoxin-sensitive site localized within the $\gamma$-aminobutyric acid A -gated chloride channel [22, 23]. Activation of $\gamma$-aminobutyric acid A receptors restrains synaptic excitation, and, therefore, the increase in AP found in EM and CM patients might be considered as a defensive mechanism aimed at limiting the enhanced neuronal excitability associated with migraine. This putative defensive mechanism might also involve $\gamma$-aminobutyric acid A receptors localized outside the blood-brain barrier. Accordingly, Moskowitz et al. have found that systemic injection of progesterone, AP, and other $\gamma$-aminobutyric acid-mimetic neurosteroids inhibits neurogenic edema in the rat meninges, and this effect is blocked by bicuculline, a $\gamma$-aminobutyric acid A receptor antagonist that is unable to cross the blood-brain barrier [24]. The reduction in DHEA (in EM and CM patients) and DHEAS (only in CM patients) levels might contribute to restrain neuronal excitation and neurogenic edema because both steroids behave as weak negative allosteric modulators or $\gamma$-aminobutyric acid A receptors [7].

Table 5 Plasma neurosteroid levels in the overall population of patients affected by chronic migraine during the headache attack and in the interictal period

\begin{tabular}{llll}
\hline & $\begin{array}{l}\text { Chronic migraine } \\
\text { during attack }(n=27)\end{array}$ & $\begin{array}{l}\text { Chronic migraine } \\
\text { no attack }(n=24)\end{array}$ & Controls \\
\hline AP $(\mathrm{ng} / \mathrm{mL})$ & $1.1 \pm 0.4^{*}$ & $1.1 \pm 0.2^{*}$ & $0.6 \pm 0.3$ \\
EAP $(\mathrm{ng} / \mathrm{mL})$ & $0.5 \pm 0.1$ & $0.4 \pm 0.2$ & $0.4 \pm 0.1$ \\
DHEA $(\mathrm{ng} / \mathrm{mL})$ & $1.4 \pm 0.9^{*}$ & $1.8 \pm 1.2^{*}$ & $5.1 \pm 3.8$ \\
DHEAS $(\mu \mathrm{g} / \mathrm{mL})$ & $1.5 \pm 1.1^{*}$ & $1.0 \pm 0.6^{*}$ & $2.7 \pm 2.0$ \\
\hline
\end{tabular}

Values are means + S.D. ${ }^{*} p<0.05$ vs. the respective control groups. No significant changes were found between values obtained during the attack and in the interictal period. Statistical analysis was performed by One-way ANOVA + Fisher's LSD (AP, $F_{2,79}=24.79 ;$ EAP, $F_{2,79}=1.43 ;$ DHEA, $F_{2,79}=18.06$; DHEAS, $\mathrm{F}_{2,79}=10.66$ )
Table 6 Plasma neurosteroid levels in patients affected by cluster headache and their healthy controls

\begin{tabular}{llll}
\hline & Cluster headache & Controls & $P$ \\
\hline AP $(\mathrm{ng} / \mathrm{mL})$ & $0.3 \pm 0.1$ & $0.7 \pm 0.2$ & $<0.01$ \\
EAP $(\mathrm{ng} / \mathrm{mL})$ & $0.4 \pm 0.1$ & $0.3 \pm 0.2$ & n.s. \\
DHEA $(\mathrm{ng} / \mathrm{mL})$ & $2.9 \pm 2.2$ & $4.3 \pm 5.0$ & n.s. \\
DHEAS $(\mu \mathrm{g} / \mathrm{mL})$ & $2.6 \pm 1.7$ & $2.2 \pm 1.7$ & n.s. \\
\hline Values are means + S.D. Statistical analysis was performed by Student's t test
\end{tabular}

Interestingly, and counterintuitively, the drop in DHEA and DHEAS levels was more substantial in CM patients, suggesting that adaptive mechanisms that reinforce $\gamma$-aminobutyric acid mediated transmission are more prominent in CM with respect to EM. Accordingly, only EM patients showed increases in the levels of EAP, a neurosteroid that is devoid of intrinsic efficacy and behaves as competitive antagonist at the AP site of $\gamma$-aminobutyric acid A receptors [20]. Perhaps, it is a high recurrence of migraneous attacks that drives the large reductions in DHEA and DHEAS (associated with the increase in AP) as an extreme but unsuccessful attempt to reinforce $\gamma$-aminobutyric acid mediated transmission. In contrast, even a lower reduction in DHEA levels (always associated with the increase in AP levels) might be sufficient to restrain migraneous episodes in patients affected by EM. Interestingly, patients affected by $\mathrm{CM}+\mathrm{MO}$ showed reduced AP levels and AP/EAP ratio during the migraneous attack, whereas the remaining $\mathrm{CM}$ patients showed increases in DHEAS levels during the attack. These findings suggest that either changes in neurosteroid levels are causally related to the onset of the migraneous attack, or, alternatively, is the attack itself that causes alterations in neurosteroid levels. We favour the former hypothesis because a reduction in AP levels (in $\mathrm{CM}+\mathrm{MO}$ patients) and an increase in DHEAS levels (in non-CM + MO patients) should restrain synaptic inhibition. Longitudinal studies in patients are necessary to establish whether measurements of plasma neurosteroids may help to predict the transition from EM to CM.

A different scenario was observed in patients affected by $\mathrm{CH}$, in which AP levels were largely reduced, and not increased as observed in EM or CM patients. This suggests that changes in neurosteroid levels in $\mathrm{CH}$ are not "defensive", but might contribute to the pathophysiology of $\mathrm{CH}$, lending credit to the hypothesis that migraine and $\mathrm{CH}$ are different disease, yet sharing an increased neuronal excitability as a common pathophysiological substrate. The reduction of AP levels could also help to explain the more severe and disabling clinical phenotype of $\mathrm{CH}$, as compared to migraine. Progesterone is a metabolic precursor of both AP and testosterone, and testosterone levels are known to be reduced in $\mathrm{CH}$ [9]. No data are available on changes in 
progesterone levels in $\mathrm{CH}$ because the disorder is highly prevalent in males. Perhaps there is a primary reduction in progesterone levels in $\mathrm{CH}$, which results into a secondary drop in both AP and testosterone levels. This interesting hypothesis warrants further investigation.

\section{Conclusion}

In conclusion, we have shown for the first time that large and disease-specific changes in circulating neurosteroid levels are associated with chronic headache disorders, raising the interesting possibility that fluctuations of neurosteroids at their site of action might shape the natural course of migraine and $\mathrm{CH}$. Whether the observed changes in neurosteroids are genetically determined or rather result from exposure to environmental or intrinsic stressors [25] is unknown. This might also be matter for further investigation because stress is a known triggering factor for headache attacks in both migraineurs and $\mathrm{CH}$ patients.

In the future, it will be interesting to verify also the correlations between specific comorbidities presenting similar neural hyperexcitability of migraine, like fibromyalgia [26], or correlate the neuroinflammation related to dyslipidemia in migraine [27].

Last, our findings may lay the groundwork for novel neurosteroid-based therapeutic strategies in the treatment chronic headache disorders. Ganaxolone, a synthetic $\gamma$-aminobutyric acid-mimetic analogue of AP, has been developed for the treatment of epilepsy, and it showed good efficacy and safety profile in patients with uncontrolled focal seizures in a phase-II placebo controlled clinical trial [28]. Our data encourage the experimental use of ganoxolone particularly in $\mathrm{CH}$ patients, where AP levels were found to be largely reduced.

\footnotetext{
Abbreviations

AP: Allopregnanolone; BDI Beck: Depression Inventory; $\mathrm{CH}$ : Cluster headache; CM: Chronic migraine; CNS: Central Nervous System; CONSORT: Consolidated Standards of Reporting Trials; DHEA: Dehydroepiandrosterone; DHEAS: Dehydroepiandrosterone sulfate; EAP: Epiallopregnanolone; EM: Episodic migraine; HIT-6: Headache impact test; ICHD3 ß: International Classification of Headache Disorders 3rd Edition B version; LC-MS/MS: Liquid chromatography tandem mass spectrometry; MIDAS: Migraine disability assessment test; $\mathrm{MOH}$ : Medication overuse headache; SAS: Self-rating anxiety scale
}

\section{Acknowledgements}

The authors wish to acknowledge and thank Prof. Alessandro Laviano for critically reviewing the manuscript before submission.

\section{Funding}

The costs of the study were in part covered by grant $n$. 00210_10_4515DMCM from Sapienza University, Rome, Italy.

All authors have read the journal's policy on disclosure of potential conflicts of interest and declare no conflict of interest.

\section{Authors' contributions}

AK collected and analyzed the data and wrote the manuscript. CC, LL and MC analyzed the samples and performed the statistical analysis. MM, MG, FP and ES collected and prepared the samples for the analysis. CMDM and MG screened the patients and collected the samples. AN analyzed the results and reviewed the manuscript. LDM performed the statistical analysis. MS analyzed the results and significantly contributed to the writing of the paper. FN reviewed the manuscript and contributed with relevant conceptual interpretations. PM designed the study, overlooked to practical implementation of the study and contributed to the writing of the manuscript. All authors read and approved the final manuscript.

\section{Ethics approval and consent to participate}

The protocol has been approved by the Azienda Ospedaliero-Universitaria Sant'Andrea Ethics Committee. All the patients gave their written informed consent to participate.

\section{Consent for publication}

All the authors approved the final draft of the manuscript.

\section{Competing interests}

The authors declare no competing financial or non-financial interests related to the content of the manuscript.

\section{Publisher's Note}

Springer Nature remains neutral with regard to jurisdictional claims in published maps and institutional affiliations.

\section{Author details}

${ }^{1}$ Department of Clinical and Molecular Medicine, Sapienza University and Regional Referral Headache Centre, Sant'Andrea Hospital, via di Grottarossa 1035-1039, 00189 Rome, Italy. ${ }^{2}$ Residency Program of Internal Medicine, School of Medicine and Psychology, Sapienza University, 00189 Rome, Italy. ${ }^{3}$ Laboratory of Advanced Molecular Diagnostics, IRCSS Istituto Dermopatico dell'Immacolata, 00167 Rome, Italy. 'Laboratory of Experimental Immunology, IRCSS Istituto Dermopatico dell'Immacolata, 00167 Rome, Italy. ${ }^{5}$ Regional Referral Headache Centre, Sant'Andrea Hospital, 00189 Rome, Italy. ${ }^{6}$ Department of Medical, Surgical and Experimental Sciences, University of Sassari, Sassari, Italy. ${ }^{7}$ IRCCS Neuromed, 86077 Pozzilli (IS), Italy. ${ }^{8}$ Department of Neurosciences, Mental Health and Sensory Organs, Sapienza University, 00189 Rome, Italy. ${ }^{9}$ Advanced Molecular Diagnostics Unit, Sant'Andrea Hospital, 00189 Rome, Italy. ${ }^{10}$ Department of Physiology and Pharmacology "Vittorio Erspamer", Sapienza University, 00189 Rome, Italy.

Received: 20 March 2019 Accepted: 24 April 2019

Published online: 27 May 2019

\section{References}

1. GBD 2015 Disease and Injury Incidence and Prevalence Collaborators (2016) Global, regional, and national incidence, prevalence, and years lived with disability for 310 diseases and injuries, 1990-2015: a systematic analysis for the Global Burden of Disease Study 2015. Lancet 388:1545-1602

2. Steiner TJ, Stovner LJ, Vos T, Jensen R, Katsarava Z (2018) Migraine is first cause of disability in under 50s: will health politicians now take notice? J Headache Pain 19:17

3. May A, Schulte LH (2016) Chronic migraine: risk factors, mechanisms and treatment. Nat Rev Neurol 12:455-464

4. Reddy DS, Estes WA (2016) Clinical potential of neurosteroids for CNS disorders. Trends Pharmacol Sci 37:543-561

5. Hosie AM, Wilkins ME, Smart TG (2007) Neurosteroid binding sites on GABAA receptors. Pharmacol Ther 116:7-19

6. Friess E, Schiffelholz T, Steckler T, Steiger A (2000) Dehydroepiandrosteronea neurosteroid. Eur J Clin Investig 30(suppl..46-50

7. Naylor JC, Kilts JD, Strauss JL, Szabo ST, Dunn CE, Wagner HR et al (2016) An exploratory pilot investigation of neurosteroids and self-reported pain in female Iraq/Afghanistan-era veterans. J Rehabil Res Dev 53:499-510

8. Taubø\|l E, Sveberg L, Svalheim S (2015) Interactions between hormones and epilepsy. Seizure 28:3-11

9. Reddy DS (2014) Neurosteroids and their role in sex-specific epilepsies. Neurobiol Dis (Pt. B) 72:198-209 
10. Pietrobon D, Striessnig J (2003) Neurobiology of migraine. Nat Rev Neurosci 5:386-398

11. Pietrobon D, Moskowitz MA (2014) Chaos and commotion in the wake of cortical spreading depression and spreading depolarizations. Nat Rev Neurosci 6:379-393

12. Aguila ME, Rebbeck T, Leaver AM, Lagopoulos J, Brennan PC, Hübscher M et al (2016) The association between clinical characteristics of migraine and brain GABA levels: an exploratory study. J Pain 17:1058-1067

13. Headache Classification Committee of the International Headache Society (IHS) (2013) The international classification of headache disorders, 3rd edition (beta version). Cephalalgia 33:629-808

14. Diener HC, Holle D, Solbach K, Gaul C (2016) Medication-overuse headache: risk factors, pathophysiology and management. Nat Rev Neurol 12:575-583

15. Martelletti P (2018) The journey from genetic predisposition to medication overuse headache to its acquisition as sequela of chronic migraine. J Headache Pain 19:2

16. Negro A, Martelletti P (2011) Chronic migraine plus medication overuse headache: two entities or not? J Headache Pain 12:593-601

17. Lionetto L, De Andrés F, Capi M, Curto M, Sabato D, Simmaco M et al (2017) LC-MS/MS simultaneous analysis of allopregnanolone, epiallopregnanolone, pregnanolone, dehydroepiandrosterone and dehydroepiandrosterone 3-sulfate in human plasma. Bioanalysis 9:527-539

18. Guennoun R, Labombarda F, Gonzalez Deniselle MC, Liere P, De Nicola AF, Schumacher M (2015) Progesterone and allopregnanolone in the central nervous system: response to injury and implication for neuroprotection. J Steroid Biochem Mol Biol 146:48-61

19. Paul SM, Purdy RH (1992) Neuroactive steroids. FASEB I 6:2311-2322

20. Wang MD, Bäckström T, Landgren S (2000) The inhibitory effects of allopregnanolone and pregnanolone on the population spike, evoked in the rat hippocampal CA1 stratum pyramidale in vitro, can be blocked selectively by epiallopregnanolone. Acta Physiol Scand 169:333-341

21. Sutherland HG, Griffiths LR (2017) Genetics of migraine: insights into the molecular basis of migraine disorders. Headache 57:537-569

22. Biagini G, Rustichelli C, Curia G, Vinet J, Lucchi C, Pugnaghi M et al (2013) Neurosteroids and epileptogenesis. J Neuroendocrinol 25:980-990

23. Belelli D, Lambert JJ (2005) Neurosteroids: endogenous regulators of the GABA(A) receptor. Nat Rev Neurosci 7:565-575

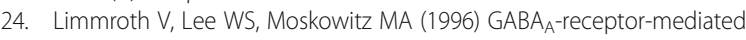
effects of progesterone, its ring-A-reduced metabolites and synthetic neuroactive steroids on neurogenic oedema in the rat meninges. Br J Pharmacol 117:99-104

25. Holsboer F, Grasser A, Friess E, Wiedemann K. Steroid effects on central neurons and implications for psychiatric and neurological disorders. Ann $N$ Y Acad Sci. 1994;746:345-59.

26. Giamberardino MA, Affaitati G, Martelletti P, Tana C, Negro A, Lapenna D, Curto M, Schiavone C, Stellin L, Cipollone F, Costantini R (2015) Impact of migraine on fibromyalgia symptoms. J Headache Pain 17:28

27. Tana C, Santilli F, Martelletti P, di Vincenzo A, Cipollone F, Davì G, Giamberardino MA (2015) Correlation between migraine severity and cholesterol levels. Pain Pract 15(7):662-670

28. Rasmusson AM, Marx CE, Pineles SL, Locci A, Scioli-Salter ER, Nillni Yl et al (2017) Neuroactive steroids and PTSD treatment. Neurosci Lett 649:156-163

Ready to submit your research? Choose BMC and benefit from:

- fast, convenient online submission

- thorough peer review by experienced researchers in your field

- rapid publication on acceptance

- support for research data, including large and complex data types

- gold Open Access which fosters wider collaboration and increased citations

- maximum visibility for your research: over $100 \mathrm{M}$ website views per year

At BMC, research is always in progress.

Learn more biomedcentral.com/submissions 